TUW 97-12

ITP-SB 97-37

hep-th/9707163

\title{
No saturation of the quantum Bogomolnyi bound by two-dimensional supersymmetric solitons
}

\author{
A. Rebhan ${ }^{1}$ and P. van Nieuwenhuizen ${ }^{2}$ \\ ${ }^{1}$ Institut für Theoretische Physik, Technische Universität Wien, \\ Wiedner Hauptstr. 8-10, A-1040 Vienna, Austria \\ ${ }^{2}$ Institute for Theoretical Physics, S.U.N.Y. at Stony Brook, \\ Stony Brook, NY 11794-3840, U.S.A.
}

\begin{abstract}
We reanalyse the question whether the quantum Bogomolnyi bound is saturated in the two-dimensional supersymmetric kink and sine-Gordon models. Our starting point is the usual expression for the one-loop correction to the mass of a soliton in terms of sums over zero-point energies. To regulate these sums, most authors put the system in a box with suitable boundary conditions, and impose an ultraviolet cut-off. We distinguish between an energy cut-off and a mode number cut-off, and show that they lead to different results. We claim that only the mode cut-off yields correct results, and only if one considers exactly the same number of bosonic and fermionic modes in the total sum over bound-state and zero-point energies. To substantiate this claim, we show that in the sine-Gordon model only the mode cut-off yields a result for the quantum soliton mass that is consistent with the exact result for the spectrum as obtained by Dashen et al. from quantising the so-called breather solution. In the supersymmetric case, our conclusion is that contrary to previous claims the quantum Bogomolnyi bound is not saturated in any of the two-dimensional models considered.
\end{abstract}




\section{Introduction and conclusion}

More than a decade ago the quantization of solitons in 1+1-dimensional models [1, 2, 3, 4, 5, 6] was extended to their supersymmetric versions [0], and the problem of how to compute the mass of a soliton at the quantum level was studied by several authors using various methods [8, 9, 10, 11, 12, 13, 14, 15, 16, 17]. With the present activity in quantum field theory regarding dualities between extended objects and pointlike particles, interest in the quantum mass of solitons has come back. In view of certain discrepancies in the above-quoted literature, we reanalyse here the question whether the Bogomolnyi bound in the supersymmetric kink and sine-Gordon model is saturated at order $\hbar$. Since in two-dimensional supersymmetry no "multiplet shortening" arises, the beautiful and simple arguments which have been used in four-dimensional $N=2$ models [18 do not apply, and a more detailed analysis seems necessary.

Our starting point is an expression for the order $\hbar$ corrections to the mass of an extended object in terms of sums over zero-point energies of fluctuations around it,

$$
M=M_{c l .}+\frac{\hbar}{2}\left(\sum \omega_{B}-\sum \omega_{B}^{\prime}\right)-\frac{\hbar}{2}\left(\sum \omega_{F}-\sum \omega_{F}^{\prime}\right)+\delta M .
$$

Here $M_{c l}$. is the classical mass expressed in terms of renormalized parameters and $\delta M$ denotes the counter-terms which are first determined in the trivial vacuum by imposing a suitable set of renormalization conditions and which we then also use in the topologically nontrivial sector. The zero-point energies are denoted by $\hbar \omega_{B, F}$ for bosons and fermions, respectively, and the vacuum part is marked by a prime. The problem is then to give a precise meaning to these infinite sums.

Most authors put the system in a large box of length $L$ with suitable boundary conditions, leading to discretized frequencies $\omega$, and then introduce an ultraviolet cut-off $\Lambda$ which restricts each sum to a finite number of terms. Then one first lets $L$ tend to infinity, and afterwards the ultraviolet cut-off is removed. There are several different boundary conditions on the fluctuations that one may impose. Various authors have demonstrated the sensitivity of the results on the boundary conditions, but (as we discuss in the Appendix) the requirement that the zero-point energies cancel mode by mode in the

trivial vacuum together with finiteness of the quantum mass of the soliton 
fixes this ambiguity.

We claim that the different ways of choosing the ultraviolet cut-off that have been used in the literature are in fact inequivalent. In particular we consider here two schemes that are frequently adopted (although often only implicitly): energy/momentum cut-off where all energies $\omega=\sqrt{k^{2}+m^{2}}$ in the continuum part of the spectrum are cut off at a value $\Lambda$, and modenumber cut-off, where in each of the four infinite sums above the same finite number $N$ of modes is retained. Since in addition to the continuum (scattering) states there are also in general bound states and zero modes, we have to specify this cut-off further. Motivated by lattice regularization [8, [1], we require the total number of these modes to be the same in each sector, which in the presence of bound states means that fewer scattering states have to be taken into account in the nontrivial sector.

Both the energy cut-off and the mode cut-off lead to finite answers for the quantum mass of the soliton, but they turn out to be mutually inconsistent, as we first show in Sect. 2 in the bosonic kink model. Some authors have tried to circumvent such issues by not considering a finite quantization volume at all [12, 13] by using e. g. general trace formulae over the energy spectrum. It seems to us that these approaches amout to a particular choice of the ultraviolet cut-off (namely an energy cut-off) and thus do not resolve the ambiguity in the procedures. Without further input one hardly can decide which procedure is the correct one. Turning to the sine-Gordon model in Sect. 3 we find a similar discrepancy, but in this case we can compare the results for the quantum soliton mass with the exact spectrum obtained by Dashen et al. from quantising the so-called breather solution. It turns out that the mode cut-off but not the energy-cutoff passes this test. We then use the same mode cut-off in a calculation of the quantum corrections to the mass of the solitons in the supersymmetric kink and sine-Gordon systems in Sect. 4. In Sect. 5 we determine the quantum corrections to the Bogomolnyi bound by expanding the central charge operator to second order in the quantum fields and computing its expectation value. The latter contains the same divergent expression that occurs in the sum over zero-point energies and can be absorbed by renormalization. Having obtained a finite result at the order $\hbar$, we can finally answer the question whether the Bogomolnyi bound is saturated by the quantized solitons. The answer is that it is not. 


\section{Quantum corrections to the kink mass}

The simplest field-theoretical model with solitons is given by a real scalar field in $1+1$ dimensions with spontaneously broken $Z_{2}$ symmetry as described by the Lagrangian

$$
\mathcal{L}=-\frac{1}{2}\left(\partial_{\mu} \varphi\right)^{2}-\frac{\lambda}{4}\left(\varphi^{2}-\mu_{0}^{2} / \lambda\right)^{2}
$$

This model has two degenerate vacuum states, $\varphi= \pm \mu_{0} / \sqrt{\lambda}$, and two static stable finite-energy (soliton) solutions, the so-called "kink" and "antikink" [1, 2]

$$
\varphi_{K, \bar{K}}= \pm \frac{\mu_{0}}{\sqrt{\lambda}} \tanh \left(\mu_{0}\left(x-x_{0}\right) / \sqrt{2}\right)
$$

with classical (unrenormalized) rest-frame energy $M_{0}=2 \sqrt{2} \mu_{0}^{3} / 3 \lambda$.

In the corresponding quantum theory we have to relate bare and renormalized parameters through appropriate counter-terms. We expand $\varphi$ about one of the vacua, $\varphi=\mu / \sqrt{\lambda}+\eta$ where $\mu_{0}^{2}=\mu^{2}+\delta \mu^{2}$. Then

$$
\mathcal{L}=-\frac{1}{2}\left(\partial_{\mu} \eta\right)^{2}-\mu^{2} \eta^{2}-\mu \sqrt{\lambda} \eta^{3}-\frac{1}{4} \lambda \eta^{4}+\frac{1}{2} \delta \mu^{2}\left(\eta^{2}+2 \frac{\mu}{\sqrt{\lambda}} \eta\right)+O\left(\hbar^{2}\right),
$$

hence the renormalized mass of the physical boson at tree-graph level is $m^{2}=2 \mu^{2}$. We fix $\delta \mu^{2}$ by requiring that the one-loop tadpole vanishes exactly, which gives

$$
\delta \mu^{2}=-3 i \lambda \hbar \int \frac{d^{2} k}{(2 \pi)^{2}} \frac{1}{k^{2}+m^{2}}=\frac{3 \lambda \hbar}{2 \pi} \int_{0}^{\Lambda} \frac{d k}{\left(k^{2}+m^{2}\right)^{1 / 2}}
$$

where we have introduced an ultraviolet cutoff $\Lambda$.

Since only the mass receives a divergent contribution, we may choose a minimal renormalization scheme defined at all loops by

$$
Z_{\lambda}=1, \quad Z_{\eta}=1, \quad \mu_{0}^{2}=\mu^{2}+\delta \mu^{2}
$$

This is the renormalization scheme that has been adopted more or less implicitly in Refs. [1, 2]. It has the advantage of maximal simplicity, but one must not forget that there are still finite corrections if one is interested in physical definitions of the various parameters. Defining for instance the physical mass of the boson through the pole of its propagator leads to an 
additional finite contribution from the self-energy diagram with 3-vertices whereas the diagram with a 4 -vertex is cancelled by (5),

$$
m_{P}^{2}=m^{2}+9 \lambda i \hbar \int \frac{d^{2} k}{(2 \pi)^{2}} \frac{m^{2}}{\left(k^{2}+m^{2}\right)\left((k-p)^{2}+m^{2}\right)}{ }_{\mid p^{2} \rightarrow-m^{2}}=m^{2}-\frac{\sqrt{3}}{2} \hbar \lambda .
$$

Evaluating the leading quantum corrections to the mass of the solitons requires the computation of the functional determinant of the differential operator describing fluctuations around the nontrivial solutions (3). This leads to [1]

$$
M=m^{3} / 3 \lambda+\frac{\hbar}{2}\left(\sum \omega-\sum \omega^{\prime}\right)+\delta M(\delta \mu)+O(\lambda)
$$

where $\omega$ and $\omega^{\prime}$ are the eigenfrequencies of fluctuations around a kink and the vacuum, respectively, and

$$
\delta M(\delta \mu)=-\frac{1}{2} \delta \mu^{2} \int_{-\infty}^{\infty}\left[\varphi_{K}^{2}(x)-\frac{m^{2}}{2 \lambda}\right] d x=\frac{m}{\lambda} \delta \mu^{2} .
$$

The latter is the contribution to the energy of the kink induced by the counterterm $\frac{1}{2} \delta \mu^{2} \varphi^{2}$ in the renormalized Lagrangian, and, as one may check, $m^{3} / 3 \lambda+\delta M(\delta \mu)=M_{0}$.

The normal modes of fluctuations around $\varphi_{K}$ are given by

$$
\left(-\frac{d^{2}}{d x^{2}}+V^{\prime \prime}\left(\varphi_{K}\right)\right) \eta_{n}(x)=\omega_{n}^{2} \eta_{n}(x)
$$

and can be expressed in terms of elementary functions [1]. Eq. (10) has two discrete eigenvalues, $\omega_{z}=0$, corresponding to a translational zero mode, and $\omega_{e}=\sqrt{3} \mathrm{~m} / 2$, which corresponds to an excited state of the kink, followed by a continuum of eigenvalues $\omega=\sqrt{k^{2}+m^{2}}$ corresponding asymptotically to waves with a $k$-dependent phase shift,

$\eta_{k}(x) \sim \exp (i[k x \pm \delta(k) / 2]) \quad$ for $x \rightarrow \pm \infty \quad$ with $\delta(k)=-2 \arctan \frac{3 m k}{m^{2}-2 k^{2}}$

In (8) the difference of the two sums has to be calculated very carefully. Both expressions are quadratically divergent in the ultraviolet, while their difference is still logarithmically divergent. It is advisable to start with a 
finite interval $x \in(-L / 2, L / 2), L \gg 1 / m$, in order to make all eigenvalues discrete so that we are actually dealing with proper sums. Choosing periodic boundary conditions , $k_{n}$ and $k_{n}^{\prime}$ can be labelled by integer numbers through the relation

$$
k_{n} L+\delta\left(k_{n}\right)=2 \pi n=k_{n}^{\prime} L,
$$

and the summation in (8) includes all integers $n$.

\section{$2.1 \quad$ Energy/momentum cutoff}

In the ultraviolet, we have already introduced a cutoff regularization when calculating the renormalized boson mass. It seems natural to also simply cut the diverging sums in (8) such that $k_{n}, k_{n}^{\prime} \leq \Lambda$. Since both in the vacuum and in the kink sector the energy of the fluctuations is given by the same $\sqrt{k^{2}+m^{2}}$, this corresponds to using the same energy cutoff for the two types of fluctuations.

In the limit $L \rightarrow \infty$, the infinite sums over $n$ can be replaced by momentum integrals using slightly different spectral densities

$$
\frac{d n}{d k}=\frac{1}{2 \pi}\left(L+\frac{d \delta(k)}{d k}\right), \quad \frac{d n}{d k^{\prime}}=\frac{L}{2 \pi} .
$$

This leads to

$$
\begin{aligned}
M_{\mathrm{EMC}} & =m^{3} / 3 \lambda+\hbar \sqrt{3} m / 4+\frac{\hbar}{2 \pi} \int_{0}^{\Lambda} d k \sqrt{k^{2}+m^{2}} \delta^{\prime}(k)+\frac{m}{\lambda} \delta \mu^{2} \\
& =m^{3} / 3 \lambda+\hbar m / 4 \sqrt{3}
\end{aligned}
$$

where we have used

$$
\frac{\hbar}{2 \pi} \int_{0}^{\Lambda} d k \sqrt{k^{2}+m^{2}} \delta^{\prime}(k)+\frac{m}{\lambda} \delta \mu^{2}=\frac{-3 m \hbar}{8 \pi} \int_{0}^{\Lambda / m} \frac{d x}{\sqrt{x^{2}+1}\left(x^{2}+\frac{1}{4}\right)}=-\frac{\sqrt{3} m \hbar}{6} .
$$

The subscript EMC refers to the strict energy or momentum cutoff that we have used here.

\footnotetext{
${ }^{1}$ Alternative boundary conditions are discussed in the Appendix.
} 


\subsection{Mode-number cutoff}

However, the result for the mass of the quantum kink as reported in the literature differs from (14). In Ref. [2], Dashen et al. have proposed to use a lattice regularization. On a lattice in a finite box, the number of degrees of freedom becomes not only countable but finite.

When comparing the contributions with and without the kink background, we have to compare mode by mode, since in both cases the number of modes is the same and every single mode, even when it belongs to the continuum eventually, makes a finite contribution. Since, in the presence of the kink, there are two discrete modes, we have to exclude two of the "continuum" modes when comparing with the energy contributions of the vacuum. Denoting by $\Delta E$ the contributions from the zero-point energies we have

$$
\begin{aligned}
\Delta E_{\mathrm{kink}} & -\Delta E_{\mathrm{vac}}=\frac{\hbar}{2} \frac{\sqrt{3} m}{2}+\frac{\hbar}{2} \sum_{-(N-1)}^{N-1} \sqrt{k_{n}^{2}+m^{2}}-\frac{\hbar}{2} \sum_{-N}^{N} \sqrt{k_{n}^{\prime 2}+m^{2}} \\
& =\frac{\hbar}{2} \frac{\sqrt{3} m}{2}-\hbar m+\hbar \sum_{n=1}^{N}\left(\sqrt{k_{n-1}^{2}+m^{2}}-\sqrt{k_{n}^{\prime 2}+m^{2}}\right)
\end{aligned}
$$

Because

$$
k_{n-1} L+\left[\delta\left(k_{n-1}\right)+2 \pi\right]=2 \pi n=k_{n}^{\prime} L,
$$

we obtain in the limit of infinite volume

$$
\Delta E_{\mathrm{kink}}-\Delta E_{\mathrm{vac}}=\frac{\hbar}{2} \frac{\sqrt{3} m}{2}-\hbar m-\hbar \int_{0}^{\Lambda} \frac{d k}{2 \pi} \frac{d \sqrt{k^{2}+m^{2}}}{d k}[\delta(k)+2 \pi] .
$$

This result differs from the corresponding result with a strict energy cutoff by the amount

$$
-\hbar m-\left.\frac{\hbar}{2 \pi} \sqrt{k^{2}+m^{2}}[\delta(k)+2 \pi]\right|_{0} ^{\Lambda \rightarrow \infty}=-\frac{3 m \hbar}{2 \pi},
$$

where we used that $\delta(k \rightarrow \infty) \rightarrow-2 \pi$. [f

\footnotetext{
${ }^{2}$ Note that the shift in the continuum modes in (15) leads to the extra term $2 \pi$ in $[\delta+2 \pi]$, which is essential to obtain a finite answer. If one does not take the shift in the continuum modes into account one gets the same result only if one (erroneously) assumes that $\delta(k \rightarrow \infty) \rightarrow 0$ [1].
} 
The mass of the quantum kink using lattice regularization in a finite box and consequently a mode-number cutoff (MNC) is therefore

$$
M_{\mathrm{MNC}}=\frac{m^{3}}{3 \lambda}+\hbar m\left(\frac{1}{4 \sqrt{3}}-\frac{3}{2 \pi}\right)
$$

or, in terms of the physical (pole) mass of the boson (7), [4]

$$
M_{\mathrm{MNC}}=\frac{m_{P}^{3}}{3 \lambda}+\hbar m_{P}\left(\frac{1}{\sqrt{3}}-\frac{3}{2 \pi}\right) .
$$

The difference between (14) and (19) can be traced to the attractive nature of the kink. Having a fixed energy cutoff, we are taking slightly more modes into account due to the negative phase shift $\delta$, to wit,

$$
L \frac{\hbar}{2 \pi} \int_{\Lambda-\left|\frac{\delta(\Lambda)+2 \pi}{L}\right|}^{\Lambda} d k \sqrt{k^{2}+m^{2}} \stackrel{L \rightarrow \infty}{\longrightarrow} \frac{\hbar}{2 \pi}|\delta(\Lambda)+2 \pi| \Lambda \stackrel{\Lambda \rightarrow \infty}{\longrightarrow} \frac{3 m \hbar}{2 \pi} .
$$

In the vacuum, there is no difference between the two procedures-both are introducing a straightforward momentum cutoff which is eventually sent to infinity. But the mass of the quantum kink (at least it's ratio with the physical boson mass $m_{P}$ ) is a physical quantity that should not depend on the particular regularization procedure.

Just from the above results one hardly can decide which procedure is more trustworthy. But in the following section we shall see that only the mode-number cutoff as introduced by a finite lattice is leading to consistent results for the spectrum of the sine-Gordon model. Indeed, when viewed from within lattice regularization, a strict energy cutoff appears to be a very unnatural procedure: in the above calculation it would have meant comparing the quantum corrections in the kink sector to those in the vacuum by taking two slightly different lattices, the one for the kink being a little bit finer, before taking the continuum limit.

\footnotetext{
${ }^{3}$ A particular physical definition of the coupling $\lambda$, e.g. through the low-energy limit of scattering amplitudes, would also modify the result further at order $\lambda^{0}$.
} 


\section{Quantization of the sine-Gordon soliton and breather solutions}

The sine-Gordon model is defined by the Lagrangian

$$
\mathcal{L}=-\frac{1}{2}\left(\partial_{\mu} \varphi\right)^{2}+\frac{m_{0}^{2}}{\gamma}[\cos (\sqrt{\gamma} \varphi)-1] .
$$

Its potential has infinitely many degenerate minima at $\varphi=2 n \pi / \sqrt{\gamma}$ and gives rise to solitons (anti-solitons) which are known in closed form,

$$
\varphi^{s, \bar{s}}(x)=\frac{4}{\sqrt{\gamma}} \arctan \left[\exp \left( \pm m_{0}\left(x-x_{0}\right)\right)\right]
$$

with classical (unrenormalized) rest frame energy $M_{s}^{0}=8 m_{0} / \gamma$.

In the corresponding quantum field theory, expanding about the vacuum $\varphi=0$, the Lagrangian for the fluctuations becomes

$$
\mathcal{L}=-\frac{1}{2}\left(\partial_{\mu} \eta\right)^{2}-\frac{1}{2} m_{0}^{2} \eta^{2}+m_{0}^{2} \frac{\gamma}{4 !} \eta^{4}-m_{0}^{2} \frac{\gamma^{2}}{6 !} \eta^{6}+\ldots .
$$

Only the seagull loops are divergent [3, [1] so that we can choose the minimal renormalization scheme defined by

$$
Z_{\eta}=1, \quad Z_{\gamma}=1, \quad m_{0}^{2}=m^{2}+\delta m^{2}, \quad \delta m^{2}=\hbar m^{2} \frac{\gamma}{4 \pi} \int_{0}^{\Lambda} \frac{d k}{\left(k^{2}+m^{2}\right)^{1 / 2}} .
$$

The complete counter-term is then [3]

$$
\delta \mathcal{L}=-\frac{m^{2}}{\gamma}\left(e^{\delta m^{2} / m^{2}}-1\right)(1-\cos (\sqrt{\lambda} \varphi)) .
$$

Because at one-loop order there is only the logarithmically divergent seagull contribution to the mass of the boson, the above renormalized mass coincides with the physical mass defined by the pole of the propagator, up to contributions of the order $\gamma^{2}$, which will not be of interest to us but can be found in Ref. [0, [6]. 


\subsection{Quantum corrections to the soliton mass}

In order to compute the quantum corrections to the soliton mass, the solutions to the analogue of eq. (10) have to be obtained, now with $\varphi^{s}$ in place of $\varphi_{K}$. This leads to a fluctuation spectrum consisting of the translational zero mode with $\omega_{z}=0$ and a continuum with $\omega=\sqrt{k^{2}+m^{2}}$, corresponding to two-dimensional waves with asymptotic phase shift'

$$
\delta(k)=2 \arctan \frac{m}{k} .
$$

Proceeding as before (but setting $\hbar=1$ in what follows), we obtain for the quantum correction to the soliton mass

$$
\Delta M_{s}^{\mathrm{EMC}}=M_{s}^{\mathrm{EMC}}-\frac{8 m}{\gamma}=\int_{0}^{\Lambda} \frac{d k}{2 \pi} \sqrt{k^{2}+m^{2}} \delta^{\prime}(k)+\frac{4}{\gamma m} \delta m^{2} \equiv 0
$$

when using a strict energy cutoff.

On the other hand, invoking lattice regularization in a finite box and comparing an equal number of modes in each sector yields

$$
\Delta M_{s}^{\mathrm{MNC}}=\frac{1}{2}\left(\left[\sum_{-N}^{-1}+\sum_{1}^{N}\right] \sqrt{k_{n}^{2}+m^{2}}-\sum_{-N}^{N} \sqrt{k_{n}^{\prime 2}+m^{2}}\right)+\frac{4}{\gamma m} \delta m^{2}
$$

where we used that in the presence of the soliton there is no solution of eqs. (12) and (27) with $n=0$. Its place is taken by the translational zero mode which does not contribute to (29). In the continuum limit we then obtain

$$
\Delta M_{s}^{\mathrm{MNC}}=-\frac{m}{2}-\int_{0}^{\Lambda} \frac{d k}{2 \pi} \frac{d \sqrt{k^{2}+m^{2}}}{d k} \delta(k)+\frac{4}{\gamma m} \delta m^{2}=-\frac{\Lambda \delta(\Lambda)}{2 \pi} \rightarrow-\frac{m}{\pi}
$$

in agreement with Refs. [2, 5, 6].

The difference between (30) and (28) is seen to have the same explanation as in the case of the kink, cf. (21).

\footnotetext{
${ }^{4}$ Full details can be found in Ref. [6].
} 


\subsection{Quantisation of the breather solution}

Besides the above soliton solutions, which are time-independent in their restframe, one also knows exact time-dependent solutions to the sine-Gordon field equations. We shall consider the famous "breather" or doublet solution,

$$
\begin{aligned}
& \varphi_{\tau}(x, t)= \frac{4}{\sqrt{\gamma}} \arctan \left(\frac{\rho \sin (2 \pi t / \tau)}{\cosh (2 \pi \rho x / \tau)}\right), \quad \frac{2 \pi}{m}<\tau<\infty, \\
& \rho \equiv \sqrt{(m \tau / 2 \pi)^{2}-1}
\end{aligned}
$$

which corresponds to a (topologically trivial) bound state of a soliton and an anti-soliton.

Classically, its mass is given by $M_{\tau}=2 M_{s} \sqrt{1-(2 \pi / m \tau)^{2}}$. In their monumental work, Dashen et al. [4 have succeeded to quantise this solution by an adaption of the semiclassical WKB method, which leads to a refined BohrSommerfeld-type quantization condition - the DHN formula for the quantum bound states.

The DHN formula for the bound state energies reads [4, 1]

$$
\begin{aligned}
& S_{c l}\left[\varphi_{\tau}\right]+S_{c t}\left[\varphi_{\tau}\right]+E_{n} \tau\left(E_{n}\right)-\frac{1}{2} \sum_{i} \nu_{i}=2 \pi n \\
& E=-\frac{d}{d \tau}\left(S_{c l}\left[\varphi_{\tau}\right]+S_{c t}\left[\varphi_{\tau}\right]-\frac{1}{2} \sum_{i} \nu_{i}\right)
\end{aligned}
$$

where $S_{c l}\left[\varphi_{\tau}\right]$ is the classical action of the breather solution per period $\tau$, $S_{c t}$ the corresponding one from the counterterms, and $\nu_{i}$ are the so-called stability angles obtained from the solutions of the linear stability equation

$$
\left(-\frac{\partial^{2}}{\partial t^{2}}+\frac{\partial^{2}}{\partial x^{2}}-V^{\prime \prime}\left[\varphi_{\tau}\right]\right) \eta_{i}(x, t)=0
$$

with

$$
\eta_{i}(x, t+\tau)=e^{i \nu_{i}} \eta_{i}(x, t) .
$$

As shown in Ref. [四, there are two solutions of (34) with vanishing stability angle and a continuum with

$$
\nu_{j}=\tau \sqrt{k_{j}^{2}+m^{2}}
$$


where

$$
L k_{j}+\delta\left(k_{j}\right)=2 \pi j, \quad \delta\left(k_{j}\right)=4 \arctan \frac{\rho(\tau)}{k \sqrt{1+\rho(\tau)^{2}}}
$$

when put in a finite box with periodic boundary conditions.

In Ref. [1] (App. C), the evaluation of the sum over stability angles was performed by means of lattice regularization in this finite box. This leads to

$$
\begin{aligned}
\Delta S\left[\varphi_{\tau}\right] & \equiv S_{c t}\left[\varphi_{\tau}\right]-\frac{1}{2} \sum_{i} \nu_{i} \\
& =-\tau\left[\sum_{i=1}^{N}\left(\sqrt{k_{i}^{2}+m^{2}}-\sqrt{k_{i}^{\prime 2}+m^{2}}\right)-m\right]+\frac{16 \pi}{\gamma m^{2}} \rho(\tau) \delta m^{2},
\end{aligned}
$$

where in counting the modes it is essential to note that $k_{1}=k_{-1}=0$ are degenerate modes. In the limit $L \rightarrow \infty$ this becomes

$$
\begin{aligned}
\Delta S\left[\varphi_{\tau}\right]^{\mathrm{MNC}} & =\tau\left[\int_{0}^{\Lambda} \frac{d k}{2 \pi} \frac{d \sqrt{k^{2}+m^{2}}}{d k} \delta(k)+m\right]+\frac{16 \pi \rho \delta m^{2}}{\gamma m^{2}} \\
& =4(\rho-\arctan (\rho))=-\frac{\gamma}{8 \pi} S_{c l}\left[\varphi_{\tau}\right] .
\end{aligned}
$$

Thus all the quantum corrections combine into a finite change of the coupling constant

$$
\gamma \rightarrow \gamma^{\prime}=\frac{\gamma}{1-\frac{\gamma}{8 \pi}}
$$

and the bound state spectrum following from the DHN formula (32) is finally given by

$$
E_{n}=\frac{16 m}{\gamma^{\prime}} \sin \left(n \gamma^{\prime} / 16\right), \quad n=1,2, \ldots<8 \pi / \gamma^{\prime} .
$$

The lowest-lying (bound) state is found to coincide with the ordinary boson of the theory, $E_{1}=m$, and there is a series of bound-states terminating at $E=16 \mathrm{~m} / \gamma^{\prime}$, which coincides with $2 M_{s}^{\mathrm{MNC}}$ obtained by quantization in the soliton sector.

Repeating this calculation with a strict energy/momentum cutoff on $\nu / \tau$ in place of a lattice cutoff gives instead

$$
\begin{aligned}
\Delta S\left[\varphi_{\tau}\right]^{\mathrm{EMC}} & =-\tau \int_{0}^{\Lambda} \frac{d k}{2 \pi} \sqrt{k^{2}+m^{2}} \delta^{\prime}(k)+\frac{16 \pi \rho \delta m^{2}}{\gamma m^{2}} \\
& =-\frac{\gamma}{8 \pi} S_{c l}\left[\varphi_{\tau}\right]-4 \rho .
\end{aligned}
$$


If taken literally, the beautiful result (41) is completely lost. However, the extra term can be regarded as a finite mass shift that would occur in addition to the replacement of $\gamma$ by $\gamma^{\prime}$. It can be absorbed by replacing $m$ by

$$
m^{\prime}=\frac{m}{1-\frac{\gamma}{8 \pi}}
$$

in $S_{c l}\left[\varphi_{\tau}\right]$, which changes (41) to

$$
E_{n}^{\mathrm{EMC}}=\frac{16 m}{\gamma} \sin \left(n \gamma^{\prime} / 16\right), \quad n=1,2, \ldots<8 \pi / \gamma^{\prime}
$$

This indeed agrees with the quantum soliton mass as calculated before with a strict energy cutoff, but now the lowest-lying state $E_{1}=m^{\prime} \neq m$. As we have remarked already, our renormalized value $m$ is the physical one as given by the pole of the boson propagator up to terms of order $\gamma^{2}$. So we have lost the possibility to make the identification of the lowest bound state with the elementary boson. Moreover, the DHN result (41) has been checked thoroughly against perturbative calculations of boson bound states [1], to which (44) no longer fits.

Actually, the DHN formula has been derived by consistently neglecting any mass renormalization except in $S_{c t}\left[\varphi_{\tau}\right]$ (cf. the discussion in Sect. III.C of Ref. [4]). We could therefore feel entitled to simply drop the extra term in (42). This indeed restores the result (41).

Now, however, the EMC result (28) for the mass of the quantum soliton no longer fits nicely to the bound state spectrum. The highest states would no longer disappear precisely when they can decay into a soliton-antisoliton pair, but already before that.

From this we conclude that the regularization scheme given by a strict energy/momentum cutoff is inadequate for calculating quantum corrections in topologically nontrivial sectors, whereas lattice regularization in a finite box leads to consistent results. 


\section{Quantum corrections to soliton masses in supersymmetric theories}

Both of the two-dimensional models that we have considered above have a supersymmetric extension given by a Lagrangian of the form [7]

$$
\mathcal{L}=-\frac{1}{2}\left[\left(\partial_{\mu} \varphi\right)^{2}+U(\varphi)^{2}+\bar{\psi} \gamma^{\mu} \partial_{\mu} \psi+U^{\prime}(\varphi) \bar{\psi} \psi\right]
$$

where $\psi$ is a Majorana spinor, $\bar{\psi}=\psi^{\mathrm{T}} C$. We shall use a Majorana representation of the Dirac matrices with $\gamma^{0}=-i \tau^{2}, \gamma^{1}=\tau^{3}$, and $C=\tau^{2}$ so that $\psi=\left(\begin{array}{l}\psi^{+} \\ \psi^{-}\end{array}\right)$with real $\psi^{+}(x, t)$ and $\psi^{-}(x, t)$.

The kink and the sine-Gordon systems are given by

$$
U_{\text {kink }}(\varphi)=\sqrt{\frac{\lambda}{2}}\left(\varphi^{2}-\mu_{0}^{2} / \lambda\right), \quad U_{\mathrm{s}-\mathrm{G}}(\varphi)=\frac{2 m_{0}}{\sqrt{\gamma}} \sin \frac{\sqrt{\gamma} \varphi}{2},
$$

respectively.

Again we choose a minimal renormalization scheme as in (6) and (25), augmented now by $Z_{\psi}=1$. Because of the additional fermionic contributions, we now have

$$
\delta m^{2}=m^{2} \frac{\gamma}{8 \pi} \int_{0}^{\Lambda} \frac{d k}{\left(k^{2}+m^{2}\right)^{1 / 2}}
$$

in the sine-Gordon case. One finds the same value for $\delta m^{2}$ from the requirement that the bosonic seagull graph for the fermionic two-point function vanishes. In fact, the complete counter-term contains now the following fermionic terms

$$
\delta \mathcal{L}_{\text {ferm. }}=-\frac{1}{2} m \bar{\psi} \psi\left(e^{\delta m^{2} / 2 m^{2}}-1\right) \cos \frac{\sqrt{\gamma} \varphi}{2}
$$

Just as in the purely bosonic case, both for the boson and the fermion the physical mass is given by $m_{P}=m$ at one-loop order.

For the supersymmetric kink system

$$
\delta \mu^{2}=\frac{\lambda}{2 \pi} \int_{0}^{\Lambda} \frac{d k}{\left(k^{2}+m^{2}\right)^{1 / 2}}
$$


and the physical mass of the boson is given by

$$
m_{P}^{2}=m^{2}-\frac{1}{\sqrt{3}} \lambda .
$$

Also the physical mass of the fermion is given by $\left(\mathbb{Z}^{\prime}\right)$, in agreement with supersymmetry.

In both models, the counterterm contribution to the soliton mass turns out to be the same when expressed in terms of the minimally renormalized boson mass, to wit,

$$
\delta M=\frac{m}{2 \pi} \int_{0}^{\Lambda} \frac{d k}{\left(k^{2}+m^{2}\right)^{1 / 2}} .
$$

In the formula for the leading quantum corrections to the soliton masses, there is now also a fermionic contribution to the sum over eigenfrequencies of fluctuations coming with a negative sign,

$$
M=M_{c l .}+\frac{1}{2}\left(\sum \omega_{B}-\sum \omega_{B}^{\prime}\right)-\frac{1}{2}\left(\sum \omega_{F}-\sum \omega_{F}^{\prime}\right)+\delta M .
$$

In the supersymmetric case, the two vacuum contributions cancel, $\sum \omega_{B}^{\prime}-$ $\sum \omega_{F}^{\prime}=0$, while the ones from the topologically nontrivial sector clearly must not do so, for they have to combine with $\delta M$ to yield a finite result. This has been pointed out first by Schonfeld [9], whereas it was overlooked in the early literature on this subject [8, 18]. As it turns out, the crux is in the slightly different boundary conditions one has to impose on bosonic and fermionic fluctuations.

The eigenvalue equations for the bosonic and fermionic normal modes read

$$
\begin{aligned}
& {\left[-\frac{d}{d x^{2}}+U^{\prime}(\varphi)^{2}+U(\varphi) U^{\prime \prime}(\varphi)\right] \eta_{n}=\omega_{B n}^{2} \eta_{n}} \\
& {\left[\gamma^{1} \frac{d}{d x}+U^{\prime}(\varphi)\right] u_{n}=i \omega_{F n} \gamma^{0} u_{n}}
\end{aligned}
$$

where we have written $\psi(x, t)=u(x) \exp \left(-i \omega_{F} t\right)$.

With our choice of the Dirac matrices, we find the following two coupled equations for $u^{ \pm}$

$$
\left[\frac{d}{d x}+U^{\prime}(\varphi)\right] u^{+}+i \omega u^{-}=0
$$




$$
\left[\frac{d}{d x}-U^{\prime}(\varphi)\right] u^{-}+i \omega u^{+}=0
$$

Acting with $\left(d / d x-U^{\prime}\right)$ on (52), eliminating $u^{-}$using (53), and substituting $\varphi^{\prime}=-U$ leads to the same second-order equation for $u^{+}$as for the bosonic fluctuations $\eta$. Hence, the bosonic and fermionic eigenvalues are the same and $u^{+} \sim \exp \left[i\left(k x \pm \frac{1}{2} \delta(k)\right]\right.$ for $x \rightarrow \pm \infty$. From (53) one then finds that $u^{-}(x) \sim \exp \left[i\left(k x \pm \frac{1}{2} \delta^{-}(k)\right]\right.$ where

$$
\delta^{-}(k)=\delta(k)+\theta(k) \equiv \delta(k)-2 \arctan \frac{m}{k}
$$

using that

$$
U^{\prime}(\varphi(+\infty))=-U^{\prime}(\varphi(-\infty))=m
$$

in both the kink and the sine-Gordon soliton background.

We now impose the boundary conditions [11]

$$
u^{ \pm}(L / 2)=u^{ \pm}(-L / 2) .
$$

If one chooses $u^{+} \sim \cos \left(k x \pm \frac{1}{2} \delta(k)\right)$, this function satisfies these boundary conditions, since it is even under $x \leftrightarrow-x$, but the corresponding $u^{-} \sim$ $\sin \left(k x \pm \frac{1}{2} \delta^{-}(k)\right)$ is odd and thus must vanish at $x= \pm L / 2$ to satisfy its boundary condition. Conversely, if $u^{-} \sim \cos \left(k x \pm \frac{1}{2} \delta^{-}(k)\right)$ and $u^{+} \sim \sin (k x \pm$ $\left.\frac{1}{2} \delta(k)\right)$, the latter has to vanish at $x= \pm L / 2$. This yields the quantization conditions

$$
k_{n}^{(+)} L+\delta\left(k_{n}^{(+)}\right)=2 \pi n \quad \text { and } \quad k_{n}^{(-)} L+\delta\left(k_{n}^{(-)}\right)+\theta\left(k_{n}^{(-)}\right)=2 \pi n
$$

The solutions obtained by $k \rightarrow-k$ are not independent ones, since $u^{-}$follows from $u^{+}$by (52), and we may restrict $k$ to be positive. Further, for $n=0$ there is no solution for $k^{(+)}$satisfying $(56)$. Hence, one half of the zero-point energy contributions from the bosonic fluctuations is cancelled by those half of the fermionic modes having the same quantization condition as the bosonic ones, except for $n=0$, where $k_{0}^{(+)}$is allowed by the boundary conditions only for the bosonic modes. This leads to

$$
M=M_{c l}+\frac{1}{2} \sum_{n \geq 0} \omega\left(k_{n}^{(+)}\right)-\frac{1}{2} \sum_{n \geq 0} \omega\left(k_{n}^{(-)}\right)+\delta M,
$$


which has been obtained also in Ref. [9] in a rather different kind of analysis directly from the supersymmetry algebra.

However, Refs. [9] and [11] disagree as concerns the numerical result for the quantum correction to the soliton mass. In Ref. [11], the two sums were found to exactly compensate with the counterterm contribution $\delta M$ so that there would be no correction to the soliton mass in a minimal renormalization scheme. [The nonzero result given in Ref. [11] is completely due to (7').] On the other hand, Schonfeld [9] has obtained a finite difference.

We believe that both results are in error. The authors of Ref. [11] (and also those of the later works of Ref. 112, 15, 13]) have implicitly used a strict energy/momentum cutoff for both sums, which in analogy to the derivation of (114) leads to

$$
M_{\mathrm{EMC}}=M_{c l}-\frac{1}{4 \pi} \int_{0}^{\Lambda} d k \sqrt{k^{2}+m^{2}} \theta^{\prime}(k)+\delta M=M_{c l}
$$

upon inserting (54) and (48).

On the other hand, starting from a lattice of finite extent, which in the previous sections we have found to be necessary in order to obtain a consistent regularization, we obtain instead

$$
\begin{aligned}
M_{\mathrm{MNC}} & =M_{c l}+\frac{1}{2} \lim _{L \rightarrow \infty} \sum_{n=1}^{N}\left(\sqrt{k_{n}^{(+) 2}+m^{2}}-\sqrt{k_{n}^{(-) 2}+m^{2}}\right) \\
& =M_{c l}+\frac{1}{4 \pi} \int_{0}^{\Lambda} d k \frac{d \omega}{d k} \theta(k)+\delta M=M_{c l}+\frac{\pi-2}{4 \pi} m
\end{aligned}
$$

for both the supersymmetric kink and sine-Gordon soliton mass. Actually, since in all these results $\delta(k)$ drops out and $\theta(k)$ is fixed by (55), the mass formula we have arrived at is universal to soliton-bearing two-dimensional supersymmetric theories.

Comparing our result (60) with Schonfeld's [9],

$$
M_{\text {Sch. }}=M_{c l}-\frac{m}{2 \pi}
$$

who also evaluates on a mode by mode basis, one finds that his different result comes from his having included one mode more in the fermionic sum than in the bosonic one (see eq. (2.45) of [9]). Restoring equality in the number of modes would make his result agree with ours, which by contrast features a positive mass correction. As we shall now discuss, a negative sign would be in conflict with the renormalized Bogomolnyi inequality. 


\section{The renormalized Bogomolnyi bound}

The supersymmetry algebra associated with models of the form (45) reads

$$
\begin{aligned}
& \left\{Q^{ \pm}, Q^{ \pm}\right\}=2 P_{\mp}, \\
& \left\{Q^{+}, Q^{-}\right\}=2 Z=2 \int_{-\infty}^{\infty} U(\varphi) \partial_{x} \varphi d x=2 \int_{\varphi(-\infty)}^{\varphi(+\infty)} U\left(\varphi^{\prime}\right) d \varphi^{\prime},
\end{aligned}
$$

where $Q=\int j^{0} d x, j^{\mu}=-(\not \partial+U) \gamma^{\mu} \psi$.

The central charge $Z$ depends, for a given model, only on $\varphi(+\infty)$ and $\varphi(-\infty)$, and is nonzero in topologically nontrivial sectors. For the invari-

ant mass squared $\mathcal{M}^{2}=P_{0}^{2}-P_{1}^{2}=\frac{1}{2}\left(P_{+} P_{-}+P_{-} P_{-}\right)$one can derive the Bogomolnyi bound [18]

$$
\mathcal{M}^{2}=\frac{1}{2}(\bar{Q} Q)^{2}+Z^{2} \geq Z^{2}
$$

which classically is saturated by the soliton solutions, $Z_{c l}=-M_{s, c l}$.

The renormalized operator $\int U d \varphi$ is given for the kink by $\sqrt{\lambda / 2}\left(\frac{1}{3} \varphi^{3}-\right.$ $\left.\frac{\mu^{2}}{\lambda} \varphi-\frac{\delta \mu^{2}}{\lambda} \varphi\right)$ with $\varphi=\varphi_{K}+\eta$. We then obtain [12]

$$
\begin{aligned}
Z & =Z_{c l}+\int_{-\infty}^{\infty} d x \frac{d}{d x}\left[\frac{1}{2} U^{\prime}\left(\varphi_{s}\right)\left\langle\eta^{2}(x)\right\rangle\right]+\delta Z \\
& =Z_{c l}+\frac{1}{2}\left[U^{\prime}(+\infty)-U^{\prime}(-\infty)\right] \frac{1}{2 \pi} \int_{o}^{\Lambda} \frac{d k}{\sqrt{k^{2}+m^{2}}}+\delta Z \equiv Z_{c l}
\end{aligned}
$$

upon using

$$
\delta Z=\sqrt{\frac{\lambda}{2}}\left(-\frac{\delta \mu^{2}}{\lambda}\right)\left[\varphi_{K}(+\infty)-\varphi_{K}(-\infty)\right]
$$

Analogously one finds $Z=Z_{c l}$ also for the supersymmetric sine-Gordon model.

Combined with the vanishing quantum correction to the soliton mass when a simple energy/momentum cutoff is used, this result was taken [12 to imply that the Bogomolnyi bound remains saturated by solitons even in the quantum theory. For higher dimensions, a proof of this saturation at the quantum level has been given by Witten and Olive [18], but it ceases to apply in two dimensions, because "multiplet shortening" does not occur. It has been conjectured by them to hold true also in two dimensions, but this 
conjecture was motivated by the results of Ref. [8] which have been corrected by Schonfeld [9] and Kaul and Rajaraman [11]. Only the latter result would indicate a continued saturation of the Bogomolnyi bound, while the former is inconsistent with the bound due to a negative soliton mass correction. However, we believe to have shown that the regularization procedures used respectively in Ref. [9] and Refs. [11, 12, 13, 15] are inconsistent. Using a well-defined lattice regularization as proposed in Refs. [2, 4] we obtain a nonzero result for the quantum correction to the soliton mass which is seen to be consistent with the inequality (64) while implying a loss of its saturation by solitons on the quantum level.

It would be very interesting to apply our methods also to the $3+1$ dimensional $N=2$ supersymmetric systems with monopoles and to reinvestigate the question of saturation of the Bogomolnyi bound there [19, 20]. Multiplet shortening only occurs if the supersymmetry algebra is not modified at the quantum level, which can be decided by an extension of our analysis.

\section{Epilogue: A failed rescue attempt}

After having come to the above conclusions, we learnt of a paper by Uchiyama [16], where our result (60) has been derived in a somewhat different manner directly from the supersymmetry algebra, but by using a mode number cutoff (without discussing the critical sensitivity on the particular UV regularization). However, the initial conclusion [16] that the quantum soliton states in two dimensions do not saturate the Bogomolnyi bound has been withdrawn in a later analysis [17]. In Ref. [17], it was proposed that physical observables such as the Hamilton operator need to be restricted to a small fraction of the finite box used in the quantization procedure in order to become less sensitive to boundary conditions.

In particular, the Hamiltonian (but only when it is used to measure the mass of the soliton state) is modified by

$$
H\left(f^{2}\right)=\int_{-L / 2}^{L / 2} d x f^{2}(x) \mathcal{H}
$$


where $\mathcal{H}$ is the Hamiltonian density and $f(x)$ is defined by

$$
f(x)= \begin{cases}0, & \text { for } L / 2 \geq|x| \geq(\ell+d) / 2 \\ (\ell / 2-|x|) / d+1 / 2, & \text { for }(\ell+d) / 2 \geq|x| \geq(\ell-d) / 2 \\ 1, & \text { for }|x| \leq(\ell-d) / 2\end{cases}
$$

where $L \gg \ell \gg d \gg 1 / m$.

This has the effect of changing all contributions proportional to $\hbar \omega_{n}$ according to (see p. 130 of [17])

$$
\hbar \omega_{n} \rightarrow \hbar \omega_{n} \frac{\int_{-L / 2}^{L / 2} d x f^{2}(x) \eta_{n}^{*}(x) \eta_{n}(x)}{\int_{-L / 2}^{L / 2} d x \eta_{n}^{*}(x) \eta_{n}(x)}
$$

where in the fermionic case one has to substitute the two-component spinor $u_{n}$ for $\eta_{n}$. As a consequence, the final result for the quantum soliton mass (60) gets replaced by [17]

$$
M_{\mathrm{U}}=M_{c l}+\lim _{d \rightarrow \infty} \lim _{\ell \rightarrow \infty} \lim _{L \rightarrow \infty} \frac{\ell-\frac{1}{3} d}{L} \frac{\pi-2}{4 \pi} m=M_{c l}
$$

(in the minimal renormalization scheme) and one thus gets rid of the extra contribution that otherwise is responsible for the loss of the saturation of the Bogomolnyi bound.

However, if this prescription is to be the correct one, one has to redo also the calculations of the quantum corrections to the nonsupersymmetric soliton masses. In the case of the ordinary kink, this has been done in App. $\mathrm{C}$ of Ref. [17]. Here the result turns out to coincide with the one we have obtained by using a strict energy/momentum cut-off, eq. (14).

It is a straightforward matter to perform the analogous calculation for the sine-Gordon model. This requires to calculate

$$
\frac{\int_{-L / 2}^{L / 2} d x f^{2}(x) \eta_{n}^{*}(x) \eta_{n}(x)}{\int_{-L / 2}^{L / 2} d x \eta_{n}^{*}(x) \eta_{n}(x)}=\frac{\left(\ell-\frac{1}{3} d\right)\left(k^{2}+m^{2}\right) / m-2}{L\left(k^{2}+m^{2}\right) / m-2}
$$

for the fluctuations around the sine-Gordon soliton, which modifies the result (30) according to

$$
M_{\mathrm{U}}=M_{c l}-\lim _{d \rightarrow \infty} \lim _{\ell \rightarrow \infty} \lim _{L \rightarrow \infty} \frac{\ell-\frac{1}{3} d}{L} \frac{m}{\pi}=M_{c l} .
$$


Here, again, we arrive at the result we had obtained with an energy/momentum cutoff, eq. (28). But in this case we are getting into the very same trouble that led us to discard the regularization by a strict energy/momentum cutoff: the quantum soliton mass no longer fits to the bound state spectrum obtained from quantization of the breather solution.

Notice however that we have been instructed to modify the Hamiltonian in its role as observable only, but not when e. g. the fluctuation spectrum is derived. We thus believe that the prescription of Ref. [17] is inconsistent and that we have to come back to the previous conclusion of a loss of saturation of the Bogomolnyi bound in two-dimensional supersymmetric theories.

\section{Appendix: Alternative boundary conditions}

In the purely bosonic case we have followed Refs. [1, 2, 4] in choosing periodic boundary conditions for the fluctuations about the solitons. An alternative is to require that instead

$$
\eta(-L / 2)=\eta(L / 2)=0
$$

This changes the quantisation relation (12) to

$$
k_{n} L+\delta\left(k_{n}\right)=\pi n=k_{n}^{\prime} L
$$

In the vacuum sector, (73) excludes the constant mode, and in the soliton sector, the solution with $n=0$. Eq. (15) has then to be replaced by

$$
\begin{aligned}
\Delta E_{\mathrm{kink}} & -\Delta E_{\mathrm{vac}}=\frac{\hbar}{2} \frac{\sqrt{3} m}{2}+\frac{\hbar}{2} \sum_{n=1}^{2 N-2} \sqrt{k_{n}^{2}+m^{2}}-\frac{\hbar}{2} \sum_{n=1}^{2 N} \sqrt{k_{n}^{\prime 2}+m^{2}} \\
& =\frac{\hbar}{2} \frac{\sqrt{3} m}{2}-\hbar m+\hbar \sum_{n=1}^{2 N}\left(\sqrt{k_{n-2}^{2}+m^{2}}-\sqrt{k_{n}^{\prime 2}+m^{2}}\right) .
\end{aligned}
$$

Now

$$
k_{n-2} L+\left[\delta\left(k_{n-2}\right)+2 \pi\right]=\pi n=k_{n}^{\prime} L,
$$

\footnotetext{
${ }^{5}$ Strictly speaking, the $n=0$ is excluded also with periodic boundary conditions, because its derivative is odd, but in the limit of large $L$ the latter vanishes exponentially fast, and we have followed Refs. 11, 2, 田 in therefore not excluding it.
} 
and the rest of the calculation turns out to be exactly the same as before. Hence, both results for the mass of the soliton are unchanged.

In the supersymmetric case we have started from the assumption that in the vacuum sector the bosonic and fermionic zero-point energies cancel exactly. In order that this holds true mode by mode, we cannot choose the boundary conditions for bosons and fermions independently. With the choice of periodic boundary conditions for the bosonic fluctuations, we have to adopt even boundary conditions for both $u^{+}$and $u^{-}$. (Antiperiodic boundary conditions for bosonic fluctuations would require odd ones for the fermionic ones.) Requiring instead that $\eta$ vanishes at the boundaries, we have to require also that either $u^{+}$or $u^{-}$vanishes at the boundaries. In Ref. [9] a further boundary condition has been considered: $\partial_{x} \eta$ and one of the spinor components vanishing at the boundaries, but this would exclude the constant mode of the spinors without excluding the constant mode of the bosonic sector, in violation of supersymmetry.

In Ref. 9

$$
u^{-}(L / 2)=u^{-}(-L / 2)=0 \quad \text { and } \quad \eta(L / 2)=\eta(-L / 2)=0 .
$$

has been selected as the appropriate boundary conditions on grounds that certain integrations by part are allowed, and in particular that the Hamiltonian is time independent: $\dot{H}=\int \partial_{x}\left[\partial_{x} \varphi \partial_{0} \varphi-\frac{1}{2} \bar{\psi} \gamma^{1} \partial_{x} \psi\right] d x=0$. However, also our set (56) allows partial integration and leads to $\dot{H}=0$. It has the virtue of being more symmetric in $u^{+}$and $u^{-}$and moreover guarantees that all boundary terms drop from the supersymmetry algebra except for the central charge [16].

With the boundary conditions (75) we even find that the standard semiclassical formula (49) does not lead to a finite result: the divergent contribution from the sum over the difference of zero-point energies is twice the required value. Alternatively, with $u^{+}$being set to zero at the boundaries, one instead finds a vanishing contribution from this sum, which likewise does not match the divergent contribution $\delta M$. Indeed, the calculations performed in Ref. [9] determine the quantum correction to the soliton mass from the expectation value of $Q^{2}$ in the soliton sector. From this a formula is obtained which differs from (75) by having $1 / 4$ instead of $1 / 2$ in front of all the sums.

We therefore conclude that with the standard semiclassical formula (49) the requirement of mode-by-mode matching in the vacuum sector and ul- 
traviolet finiteness in the soliton sector restricts the choice to either periodic bosonic and even fermionic fluctuations or antiperiodic bosonic and odd fermionic ones. In conformity with most of the literature we have adopted the former in the present paper.

\section{Acknowledgments}

AR wishes to thank Harald Skarke for useful discussions.

\section{References}

[1] R. Rajaraman, Solitons and Instantons (North-Holland, Amsterdam, 1982)

[2] R. F. Dashen, B. Hasslacher and A. Neveu, Phys. Rev. D10 (1974) 4130

[3] S. Coleman, Phys. Rev. D11 (1975) 2088

[4] R. F. Dashen, B. Hasslacher and A. Neveu, Phys. Rev. D12 (1975) 3424

[5] H. J. De Vega, Nucl. Phys. B115 (1976) 411

[6] J. Verwaest, Nucl. Phys. B123 (1977) 100

[7] P. di Vecchia and S. Ferrara, Nucl. Phys. B130 (1977) 93; J. Hruby, Nucl. Phys. B131 (1977) 275

[8] A. d'Adda and P. di Vecchia, Phys. Lett. 73B (1978) 162; A. d'Adda, R. Horsley and P. di Vecchia, Phys. Lett. 76B (1978) 298; R. Horsley, Nucl. Phys. B151 (1979) 399

[9] J. F. Schonfeld, Nucl. Phys. B161 (1979) 125

[10] S. Rouhani, Nucl. Phys. B182 (1981) 462.

[11] R. K. Kaul and R. Rajaraman, Phys. Lett. 131B (1983) 357

[12] C. Imbimbo and S. Mukhi, Nucl. Phys. B247 (1984) 471 
[13] H. Yamagishi, Phys. Lett. 147B (1984) 425

[14] A. Uchiyama, Nucl. Phys. B244 (1984) 57

[15] A. K. Chatterjee and P. Majumdar, Phys. Rev. D30 (1984) 844; Phys. Lett. 159B (1985) 37

[16] A. Uchiyama, Prog. Theor. Phys. 75 (1986) 1214

[17] A. Uchiyama, Nucl. Phys. B278 (1986) 121

[18] E. Witten and D. Olive, Phys. Lett. 78B (1978) 97

[19] R. K. Kaul, Phys. Lett. 143B (1984) 427

[20] C. Imbimbo and S. Mukhi, Nucl. Phys. B249 (1985) 143 